\title{
Overexpression of cofilin correlates with poor survival in breast cancer: A tissue microarray analysis
}

\author{
YUSUFU MAIMAITI ${ }^{1,2^{*}}$, JIE TAN $^{1 *}$, ZEMING LIU $^{1}$, YAWEN GUO $^{1}$, YU YAN $^{1}$, \\ XIU NIE ${ }^{3}$, BANGXING HUANG $^{3}$, JING ZHOU ${ }^{1}$ and TAO HUANG ${ }^{1}$ \\ ${ }^{1}$ Department of Breast and Thyroid Surgery, Union Hospital, Tongji Medical College, Huazhong University of Science and \\ Technology, Wuhan, Hubei 430022; ${ }^{2}$ Department of General Surgery, Research Institute of Minimally Invasive, People's \\ Hospital of Xinjiang Uygur Autonomous Region, Urumqi, Xinjiang 830000; ${ }^{3}$ Department of Pathology, Union Hospital, \\ Tongji Medical College, Huazhong University of Science and Technology, Wuhan, Hubei 430022, P.R. China
}

Received September 19, 2015; Accepted April 28, 2017

DOI: $10.3892 / \mathrm{ol} .2017 .6413$

\begin{abstract}
Cofilin, a key regulator of actin cytoskeleton dynamics, is considered to be involved in cellular migration, tumor invasion and mitosis, and its activity is increased in cancer cells. To address the association between cofilin and breast cancer prognosis, which is unclear at present, cofilin expression was analyzed in tissue microarrays of tumors from 310 patients with breast cancer via immunohistochemistry. In a multivariate Cox regression analysis, a high expression of cofilin in tumor cells correlated significantly with shorter overall survival (hazard ratio, 2.22; 95\% confidence interval, 1.35-3.66, $\mathrm{P}=0.002$, and with the Nottingham histologic grade, Ki-67 status and human epidermal growth factor receptor 2 status $(\mathrm{P}=0.031,0.001$, and 0.001 , respectively). Cofilin expression was not observed as correlated with estrogen or progesterone receptor expression, tumor size or lymph node status. These data demonstrate that cofilin is associated with poor outcome, thereby suggesting that it is a potential prognostic factor in breast cancer.
\end{abstract}

Correspondence to: Professor Tao Huang or Dr Jing Zhou, Department of Breast and Thyroid Surgery, Union Hospital, Tongji Medical College, Huazhong University of Science and Technology, 1277 Jiefang Avenue, Jianghan, Wuhan, Hubei 430022, P.R. China E-mail: huangtaowh@163.com

E-mail: drtinazhou@gmail.com

${ }^{*}$ Contributed equally

Abbreviations: ADF, actin-depolymerizing factor; ER, estrogen receptor; FISH, fluorescence in situ hybridization; Her2, human epidermal growth factor receptor 2; HR, hazard ratio; IHC, immunohistochemistry; NHG, Nottingham histological grade; PR, progesterone receptor

Key words: breast cancer, cofilin, tissue array, survival, motility, actin

\section{Introduction}

Cancer cell migration and invasion account for the majority of cancer-associated mortalities (1). Increased motility of cancer cells underlies the processes of migration and invasion $(2,3)$ and is an essential step in breast cancer metastasis (4). Targeting tumor cell motility is a potential antitumor strategy $(5,6)$. In response to migratory and chemotactic stimuli, cancer cells form membrane protrusions, which initiate the multi-step migration process. Membrane protrusions result from localized polymerization of sub-membrane actin and consequent formation of actin filaments and the actin framework is widely accepted as the engine driving cell motility; several actin-binding proteins regulate the assembly and disassembly of actin filaments, and thus the dynamic behavior of the actin cytoskeleton (7-9). Of these, the ubiquitous protein cofilin is the most important effector of actin polymerization and depolymerization, generating free barbed ends via pointed-end depolymerization and filament severing $(10,11)$.

The actin-depolymerizing factor (ADF)/cofilin family includes ADF, cofilin and other proteins with similar biochemical activities. Unicellular organisms such as yeasts usually express only one ADF/cofilin isoform, whereas multicellular organisms typically express several. In certain cultured mammalian cell lines and invasive mammary tumor cells, cofilin-1 is the most abundant isoform (12), whereas $\mathrm{ADF}$ is expressed at much lower levels. In the present study, cofilin refers to cofilin-1.

Previous studies have suggested that cofilin activity correlates with cancer progression and cancer cell migration and invasion; local activation of cofilin via uncaging induces lamellipodia formation and determines the direction of cell movement (13). siRNA-mediated depletion of cofilin in carcinoma cells inhibits cell motility (12) and the assembly and stability of invadopodia and, consequently, cell invasion (14). Cofilin overexpression increases the rate of cell migration in human glioblastoma cultures (15) and pancreatic cancer (16), and correlates with poor prognosis in human pulmonary adenocarcinoma, gastric cancer, epithelial ovarian cancer and gallbladder carcinoma (17-20). Spontaneous overexpression of cofilin has been detected in invasive subpopulations 
of mammary tumor cells (21), and is directly associated with the invasion, intravasation and metastasis of mammary tumors (22). Tissue microarray analysis has demonstrated that cofilin staining positively correlates with breast tumor grade (23).

However, to the best of our knowledge there is no direct evidence implicating deregulated cofilin expression in breast cancer prognosis at present. The present study analyzed cofilin expression in tissue microarrays of tumors from 310 patients with breast cancer via immunohistochemistry (IHC). These data provide insight into the role of cofilin in invasive breast cancer and establish correlations between cofilin expression and clinical and pathological parameters.

\section{Materials and methods}

Patient material and immunostaining in breast cancer tissue microarrays. Tissue arrays containing samples of invasive breast tumors from 310 patients were purchased from the National Engineering Center for BioChips in Shanghai, China. To prepare the arrays, a $1.5 \mathrm{~mm}$ core of tumor tissue was removed from each tumor. Tumors were formalin-fixed for at least $24 \mathrm{~h}$ and paraffin-embedded. Cores were taken from the peripheral aspect of the tumor, and necrotic tissue was avoided.

The expression of cofilin, estrogen receptor (ER), progesterone receptor (PR), Ki-67, and human epidermal growth factor receptor 2 (Her2) was determined in the arrays via IHC, using the BenchMark ULTRA system (Ventana Medical Systems, Inc., Tucson, AZ, USA) and Leica BOND-MAX system (Leica Microsystems, Ltd., Milton Keynes, UK) according to the manufacturer's protocol. Normal goat serum (10\%; Boster Biological Technology, Ltd., Wuhan, China) was used as blocking reagent, and samples were blocked for $20 \mathrm{~min}$ at room temperature. UltraView Universal HRP multimer in the DAB Detection Kit (cat. no. 760-500; Ventana Medical Systems, Inc.) was used as the secondary antibody at a ready-to-use dilution and incubated for $30 \mathrm{~min}$ at $37^{\circ} \mathrm{C}$. For cofilin expression, the cofilin-specific antibody from Abcam (cat. no. ab42824; Cambridge, UK) was used at a 1:1,500 dilution and the incubation time was $8 \mathrm{~min}$ at room temperature, while all other primary antibodies required $20 \mathrm{~min}$ at $37^{\circ} \mathrm{C}$. ER and PR were demonstrated using SP1 (cat. no. 790-4325) and 1E2 (cat. no. 790-4296; both from Ventana Medical Systems, Inc.) antibodies, respectively at a ready-to-use dilution according to the protocol of the manufacturer. Negative expression was defined as $<10 \%$ positive nuclei (24). Ki-67 was demonstrated using MM1 (cat. no. PA0410; Novocastra; Leica Microsystems, Ltd.) at a ready-to-use dilution according to the protocol of the manufacturer, and the expression was considered positive ( $>14 \%$ immunostained nuclei) or negative ( $\leq 14 \%$ immunostained nuclei). Her2 expression was assessed semiquantitatively by using a standard protocol (HercepTest; Dako; Agilent Technologies, Inc., Santa Clara, CA, USA) (25) and separated into 4 grades (from 0 to $3+$ ).

Fluorescence in situ hybridization (FISH) analysis was performed in Her2 2+ samples, using the PathVysion HER-2 DNA Probe kit (Abbott Pharmaceutical Co., Ltd., Lake Bluff, IL, USA) according to the manufacturer's protocol. Her2 expression was designated as weak (IHC grade $0-1+$ or $\mathrm{FISH}^{-}$) or strong (IHC grade 3+ or $\mathrm{FISH}^{+}$). Lymph node metastasis was staged according to the American Joint Committee on Cancer TNM system (26). Ethical approval for the present study was granted by the Human Research Ethics Committee of the Taizhou Hospital of Zhejiang Province.

Scoring, evaluation and statistical analysis. IHC staining was evaluated by two experienced pathologists blinded to the clinical information. Cofilin staining intensity in the cytoplasm of tumor cells was graded $0-3$. The percentage of cofilin-positive cells was scored 0-4 (0-5, 6-25, 26-50, 51-75 and $76-100 \%$, respectively). The final cofilin expression score ranged between 0 and 3 and was based on sum of the intensity and percent positive scores (0-1, 2-3, 4-5 and 6-7, respectively; Fig. 1). The slides were scanned using an Aperio ScanScope slide scanner, and images of representative areas were captured using Image Scope software version 9.0 (Aperio Technologies, Ltd, Oxford, UK) followed by Adobe Illustrator version 16.0 (Adobe Systems, San Jose, CA, USA) (27).

Distributions of pathological and clinical parameters [age, tumor size, Nottingham histological grade (NHG)](28), and lymph node, ER, PR, Her2, and Ki-67 status) according to the final cofilin score were calculated using a one-way ANOVA or the Pearson $\chi^{2}$ test, as indicated in Table I. Multiple comparisons between the groups was performed using the Student-Newman-Keuls method. Kaplan-Meier analysis and the Breslow test were used to estimate the effect of high cofilin expression on overall survival. For the Kaplan-Meier analysis, final cofilin scores were analyzed in terms of weak (scores of 0,1 , and 2) and strong (a score of 3) expression. Cox regression proportional hazards models were used to estimate hazard ratios (HRs) for mortality from breast cancer according to cofilin expression in univariate and multivariate analyses. The covariates with $\mathrm{P}<0.05$ in the univariate analysis (lymph node, ER, and PR status) were included in the multivariate analysis. All statistical tests were two-sided, and $\mathrm{P}<0.05$ were considered to indicate a statistically significant difference. All calculations were performed using SPSS Statistics version 19 software (IBM Corp., Armonk, NY, USA).

\section{Results}

Cofilin expression is associated with clinicopathological variables. The association between cofilin staining intensity and several clinical parameters [age, tumor size, NHG, lymph node metastasis, and ER, PR, Ki-67, and Her2 expression] was determined (Table I). There was a significant association between cofilin staining intensity and $\mathrm{NHG}(\mathrm{P}=0.030)$. A trend toward a higher NHG in tumors with higher cofilin scores was observed. No tumors exhibited a cofilin score of 3 in combination with the lowest NHG score.

Cofilin expression was also associated with Her2 expression $(\mathrm{P}<0.001)$. The distribution of Her2-positive tumors paralleled the distribution of cofilin scores: The majority of Her2-positive tumors exhibited cofilin scores of 2 or 3. A similar association between Ki-67 expression and cofilin expression was observed $(\mathrm{P}=0.001)$. As Ki67 positive tumors exhibited a larger percentage of cells with high cofilin expression compared with low cofilin expression, it was hypothesized that positive Ki67 status was associated with high cofilin expression. Cofilin 

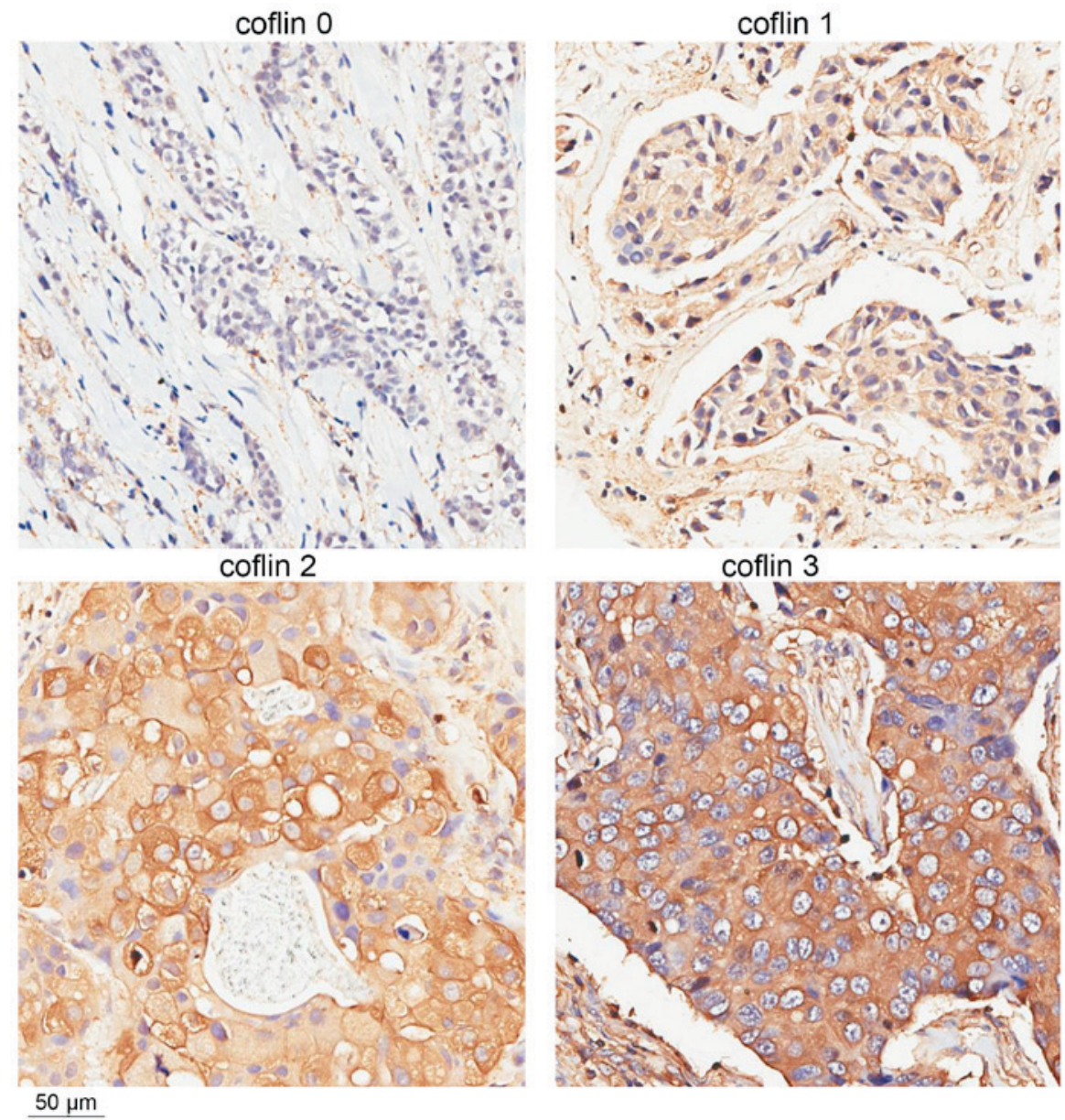

Figure 1. Examples of scoring of immunohistochemistry staining of cofilin expression in breast cancer tissue. Numbers refer to staining intensity.

expression was not significantly associated with age, tumor size, lymph node metastasis or ER or PR expression $(\mathrm{P}=0.055$, $0.294,0.082,0.084$ and 0.176 , respectively).

High expression of cofilin is associated with poor survival. For survival analysis, the cofilin scores were dichotomized: Scores of 0,1 and 2 denoted weak expression, and a score of 3 denoted strong expression. The rationale for this grouping was the marked difference in cofilin staining intensity between scores 2 and 3 (Fig. 1) and the similarity of the survival curves for patients with scores of 0,1 , or 2 (Fig. 2). Kaplan-Meier analysis demonstrated significant differences in overall survival between patients bearing tumors with weak vs. strong cofilin expression ( $\mathrm{P}=0.002$; Fig. 2). Univariate and multivariate Cox regression analyses of survival in association with cofilin expression were performed using the same dichotomized variable as in the Kaplan-Meier analysis. The results demonstrated that strong cofilin expression was an independent indicator of reduced overall survival $(\mathrm{P}=0.002$; HR, 2.22; 95\% confidence interval, 1.35-3.66) when the variables described in Table II were included. Detailed results of the Cox analyses are presented in Table II.

\section{Discussion}

Actin is the major component of the cytoskeleton, which serves an important role in tumor cell migration, invasion and mitosis. The actin-binding protein cofilin, a member of the $\mathrm{ADF} / \mathrm{cofilin}$ family, is a key regulator of actin polymerization and depolymerization. The activity and output of the cofilin pathway (cofilin and its regulatory proteins) are increased in cancer cells $(4,29,30)$. Cofilin is thought to contribute to at least 3 cancer-associated events: Initial cell transformation (31), increased cell motility during metastasis and cell division (32).

Previous studies have demonstrated that tumors with a higher NHG typically exhibited reduced tubule formation, nuclear atypia and mitoses, and Her2 expression has been associated with tumor cell proliferation and an aggressive phenotype (33-35). In the present study, cofilin staining was associated with NHG, Her2 expression and Ki-67 expression, suggesting that cofilin may be a marker of poor differentiation and high proliferation. In migrating or invading cells, cofilin resides in cell membrane protrusions, for example lamellipodia, invadopodia, and filopodia, which initiate cell movement and determine cell polarity $(36,37)$. This localization is critical for cell movement, endocytosis and cell division, all of which are important for normal cell proliferation, differentiation and cancer development (38). This promotion may be responsible for the positive association between cofilin expression and NHG, Her2 and Ki-67 status.

In agreement with previous studies, the present study identified that cofilin expression did not correlate with ER or PR status (23). There was also no correlation observed between cofilin expression and tumor size. In contrast, another study 
Table I. Associations between cofilin expression and clinicopathological features in breast cancer.

\begin{tabular}{|c|c|c|c|c|c|c|}
\hline \multirow[b]{2}{*}{ Factor } & \multirow[b]{2}{*}{ Number } & \multicolumn{4}{|c|}{ Cofilin staining intensity } & \multirow[b]{2}{*}{ P-value } \\
\hline & & 0 & 1 & 2 & 3 & \\
\hline All, n (\%) & 310 & $53(17)$ & $114(37)$ & $92(30)$ & $51(16)$ & \\
\hline Age, years $^{\mathrm{a}}$ & $54(29-88)$ & $50.5(29-83)$ & $54(31-88)$ & $57(31-87)$ & $56(37-88)$ & $0.055^{\mathrm{b}}$ \\
\hline Tumor size, $\mathrm{mm}^{\mathrm{a}}$ & $30(10-150)$ & $30(10-100)$ & $30(14-130)$ & $30(10-150)$ & $35(10-100)$ & $0.294^{\mathrm{b}}$ \\
\hline NHG, n (\%) & & & & & & $0.030^{\mathrm{d}}$ \\
\hline I & $19(6)$ & $6(32)$ & $8(42)$ & $5(26)$ & $0(0)$ & \\
\hline II & $210(68)$ & $36(17)$ & $83(40)$ & $61(29)$ & $30(14)$ & \\
\hline III & $70(22)$ & $10(14)$ & $21(30)$ & $19(27)$ & $20(29)$ & \\
\hline Missing & $11(4)$ & & & & & \\
\hline Nodal status, n (\%) & & & & & & $0.082^{\mathrm{c}}$ \\
\hline N0 & $141(45)$ & $21(15)$ & $48(34)$ & $49(34)$ & $23(16)$ & \\
\hline N1 & $86(28)$ & $14(16)$ & $33(38)$ & $21(24)$ & $18(21)$ & \\
\hline N2 & $56(18)$ & $11(20)$ & $26(46)$ & $13(23)$ & $6(11)$ & \\
\hline N3 & $21(7)$ & $7(33)$ & $2(10)$ & $8(38)$ & $4(19)$ & \\
\hline Missing & $6(2)$ & & & & & \\
\hline ER status, n (\%) & & & & & & $0.084^{c}$ \\
\hline Positive & $191(62)$ & $26(14)$ & $76(40)$ & $62(32)$ & $27(14)$ & \\
\hline Negative & $114(37)$ & $24(21)$ & $36(32)$ & $30(26)$ & $24(21)$ & \\
\hline Missing & $5(2)$ & & & & & \\
\hline PR status, $\mathrm{n}(\%)$ & & & & & & $0.176^{\mathrm{c}}$ \\
\hline Positive & $139(45)$ & $21(15)$ & $56(40)$ & $45(32)$ & $17(12)$ & \\
\hline Negative & $168(54)$ & $31(18)$ & $56(33)$ & $47(28)$ & $34(20)$ & \\
\hline Missing & $3(1)$ & & & & & \\
\hline Ki67 status, n (\%) & & & & & & $0.001^{\mathrm{c}}$ \\
\hline$>14 \%$ & $99(32)$ & $11(11)$ & $29(29)$ & $32(32)$ & $27(27)$ & \\
\hline$\leq 14 \%$ & $211(68)$ & $42(20)$ & $85(40)$ & $60(28)$ & $24(13)$ & \\
\hline Missing & $0(0)$ & & & & & \\
\hline HER 2 status, $\mathrm{n}(\%)^{\mathrm{e}}$ & & & & & & $0.000^{\mathrm{d}}$ \\
\hline Strong & $77(25)$ & $2(2)$ & $29(38)$ & $28(36)$ & $18(23)$ & \\
\hline Weak & $233(75)$ & $51(22)$ & $85(36)$ & $64(27)$ & $33(14)$ & \\
\hline Missing & $0(0)$ & & & & & \\
\hline
\end{tabular}

${ }^{\mathrm{a} D a t a}$ presented as mean (range). ${ }^{\mathrm{b}}$ One-factor analysis of variance. ${ }^{\mathrm{c}} \mathrm{Pearson} \chi^{2}$ test, 2-tailed P-value. ${ }^{\mathrm{d}}$ Fisher's excat test. ${ }^{\mathrm{e}} \mathrm{Weak}$ (score 0 - 1 , or $\mathrm{FISH}^{-}$), strong (score 3, or $\mathrm{FISH}^{+}$). NGH, Nottingham histological grade; FISH, fluorescence in situ hybridization.

demonstrated a positive association between cofilin expression and tumor stages T0, T1, and T2 (but not T3) in breast cancer (39). Resolution of this discrepancy requires additional study.

Owing to its effects on actin polymerization/depolymerization, cofilin overexpression has been associated with mammary tumor invasion, intravasation, metastasis, lymph node metastasis and a higher nodal stage. Studies on other human malignant tumor types support these associations $(20,40,41)$. However, in the present study, cofilin expression did not correlate with the nodal stage. The present study demonstrated that cofilin expression and the nodal stage are independent prognosis factors in breast cancer. As the number of positive lymph nodes largely depends on the completeness of axillary lymph node dissection, its approximation may not always be accurate (42).
Additionally, the time interval between tumor diagnosis and surgery may affect the nodal stage (43). Consequently, the nodal stage may not reflect a tendency for lymphatic metastasis. These considerations may explain why cofilin expression does not necessarily correlate with the clinical nodal stage. Active cofilin comprises only part of the total level of cofilin in the cytoplasm. The present study measured total cofilin abundance instead of cofilin activity, which is difficult to estimate. Therefore, intensive studies are needed to determine whether cofilin expression or activity is associated with nodal metastasis in human breast cancer.

Notably, high cofilin expression was significantly associated with shorter overall survival. This association remained significant when other clinicopathological factors were included in the COX regression analysis, suggesting that 
Table II. Univariate and multivariate COX regression analyses of the effects on overall survival for different patients and characteristics.

Univariate

\begin{tabular}{lcccc}
\cline { 3 - 5 } A, Variable & $\mathrm{n}$ & HR & 95\% CI & P-value \\
\hline Cofilin (strong vs. weak) $^{\mathrm{a}}$ & 310 & 2.15 & $1.32-3.49$ & 0.002 \\
ER (positive vs. negative) $_{\text {PR (positive vs. negative) }}$ & 305 & 0.63 & $0.41-0.96$ & 0.030 \\
Her2 (strong vs. weak) & 307 & 0.59 & $0.39-0.92$ & 0.019 \\
Ki67 (positive vs. negative) & 310 & 1.37 & $0.87-2.16$ & 0.177 \\
Size (>20 mm vs. $\leq 20$ mm) & 310 & 1.22 & $0.85-2.45$ & 0.374 \\
Nottingham histological grade (1-3) & 304 & 1.45 & $0.83-1.94$ & 0.173 \\
Nodal status (N3, N2, N1, N0) & 299 & 1.27 & $1.12-1.71$ & 0.269
\end{tabular}

\begin{tabular}{lccc}
\hline & \multicolumn{2}{c}{ Multivariate } \\
\cline { 2 - 4 } B, Variable & $\mathrm{n}$ & HR & 95\% CI \\
\hline Cofilin (strong vs. weak) & 299 & 2.22 & $1.35-3.66$ \\
ER (positive vs. negative) & 299 & 0.80 & $0.47-1.36$ \\
PR (positive vs. negative) & 299 & 0.75 & $0.43-1.32$ \\
Nodal status (N3, N2, N1, N0) & 299 & 1.40 & $1.13-1.73$ \\
\hline
\end{tabular}

${ }^{a}$ Cofilin score was dichotomized into weak (0-2) and strong (3). HR, hazard ratio; CI, confidence interval; ER, estrogen receptor; PR, progesterone receptor.
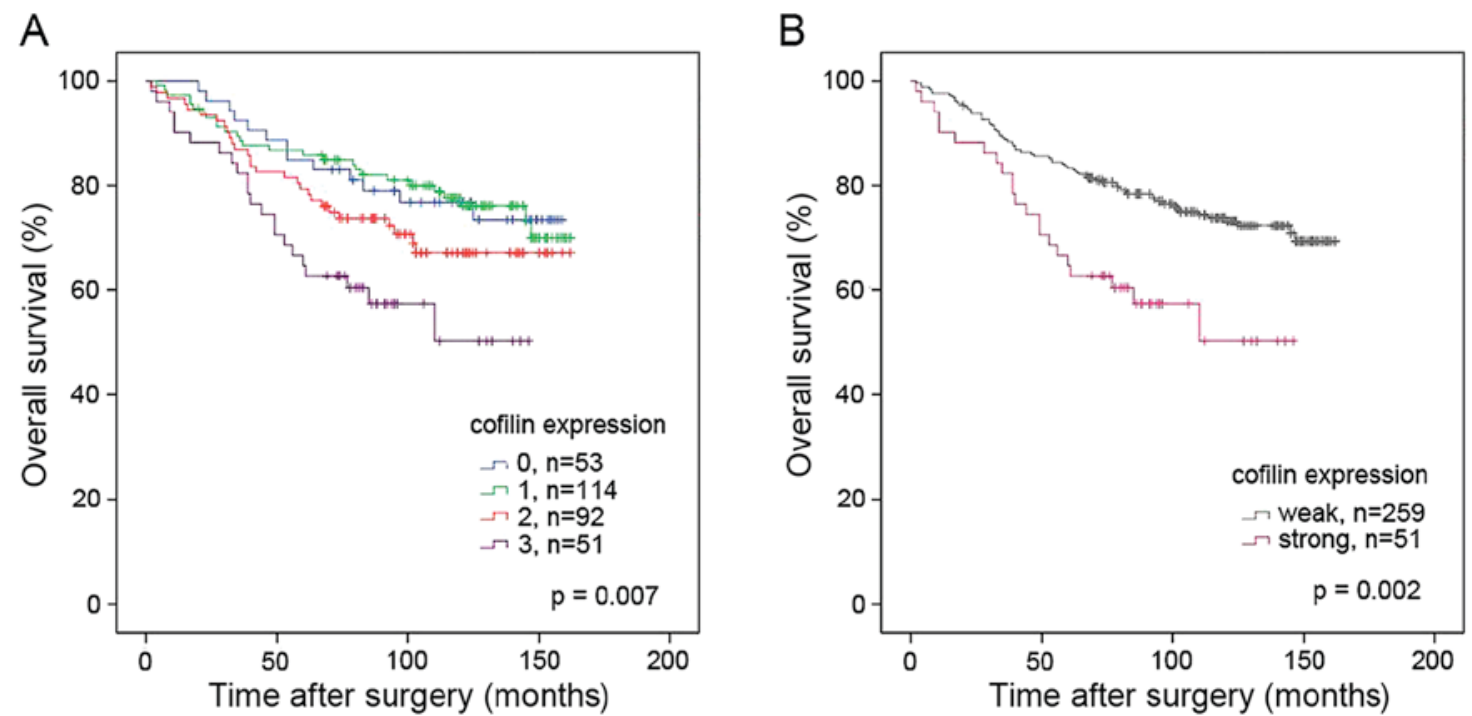

Figure 2. Effects of cofilin expression on overall survival. (A) Kaplan-Meier estimates of survival for cofilin expression demonstrate that higher expression of cofilin in tumor tissue was significantly associated with shorter overall survival. (B) Kaplan-Meier estimates of survival for weak (scores 0 , 1, and 2) vs. strong (score 3) cofilin expression in tumor tissue.

cofilin is a potential independent prognostic factor in breast cancer.

In summary, the results of the present study suggest that cofilin may promote the occurrence and development of breast cancer, perhaps via its contribution to cell migration, invasion and/or mitosis. How it does so is beyond the scope of the present study, and requires additional study. The present study suggests that cofilin is a potential independent prognostic factor in breast cancer, and raises the possibility of targeting cofilin for more effective treatment of breast cancer.

\section{Acknowledgements}

The present study was supported by the National Natural Science Foundation of China (grant no. 81001171) and the Key Technologies R\&D Program of Hubei Province 
(grant no. 2007AA302B07). The authors would like to thank Editage (https://www.editage.com/) for English language editing.

\section{References}

1. GuanX: Cancer metastases: Challenges and opportunities. Acta Pharm Sin B 5: 402-418, 2015

2. Roussos ET, Condeelis JS and Patsialou A: Chemotaxis in cancer. Nat Rev Cancer 11: 573-587, 2011.

3. Sidani M, Wessels D, Mouneimne G, Ghosh M, Goswami S, Sarmiento C, Wang W, Kuhl S, El-Sibai M, Backer JM, et al Cofilin determines the migration behavior and turning frequency of metastatic cancer cells. J Cell Biol 179: 777-791, 2007.

4. Wang W, Eddy R and Condeelis J: The cofilin pathway in breast cancer invasion and metastasis. Nat Rev Cancer 7: 429-440, 2007.

5. Moretti RM, Montagnani Marelli M, Mai S and Limonta P: Gonadotropin-releasing hormone agonists suppress melanoma cell motility and invasiveness through the inhibition of $\alpha 3$ integrin and MMP-2 expression and activity. Int J Oncol 33: 405-413, 2008.

6. Limame R, de Beeck KO, Van Laere S, Croes L, De Wilde A, Dirix L, Van Camp G, Peeters M, De Wever O, Lardon F and Pauwels P: Expression profiling of migrated and invaded breast cancer cells predicts early metastatic relapse and reveals Krüppel-like factor 9 as a potential suppressor of invasive growth in breast cancer. Oncoscience 1: 69-81, 2013

7. Carlier MF, Ressad F and Pantaloni D: Control of actin dynamics in cell motility. Role of ADF/cofilin. J Biol Chem 274: 33827-33830, 1999.

8. Achard V, Martiel JL, Michelot A, Guérin C, Reymann AC, Blanchoin L and Boujemaa-Paterski R: A 'primer'-based mechanism underlies branched actin filament network formation and motility. Curr Biol 20: 423-428, 2010.

9. Bugyi B and Carlier MF: Control of actin filament treadmilling in cell motility. Annu Rev Biophys 39: 449-470, 2010.

10. Bernstein BW and Bamburg JR: ADF/cofilin: A functional node in cell biology. Trends Cell Biol 20: 187-195, 2010.

11. Bravo-Cordero JJ, Magalhaes MA, Eddy RJ, Hodgson L and Condeelis J: Functions of cofilin in cell locomotion and invasion. Nat Rev Mol Cell Biol 14: 405-415, 2013.

12. Hotulainen P, Paunola E, Vartiainen MK and Lappalainen P. Actin-depolymerizing factor and cofilin-1 play overlapping roles in promoting rapid F-actin depolymerization in mammalian nonmuscle cells. Mol Biol Cell 16: 649-664, 2005.

13. Ghosh M, Song X, Mouneimne G, Sidani M, Lawrence DS and Condeelis JS: Cofilin promotes actin polymerization and defines the direction of cell motility. Science 304: 743-746, 2004.

14. Yamaguchi H, Lorenz M, Kempiak S, Sarmiento C, Coniglio S, Symons M, Segall J, Eddy R, Miki H, Takenawa T and Condeelis J: Molecular mechanisms of invadopodium formation: The role of the N-WASP-Arp $2 / 3$ complex pathway and cofilin. J Cell Biol 168: 441-452, 2005.

15. Yap CT, Simpson TI, Pratt T, Price DJ and Maciver SK: The motility of glioblastoma tumour cells is modulated by intracellular cofilin expression in a concentration-dependent manner. Cell Motil Cytoskeleton 60: 153-165, 2005.

16. Wang Y, Kuramitsu Y, Kitagawa T, Baron B, Yoshino S, Maehara S, Maehara Y, Oka M and Nakamura K: Cofilinphosphatase slingshot-1L (SSH1L) is over-expressed in pancreatic cancer (PC) and contributes to tumor cell migration. Cancer Lett 360: 171-176, 2015.

17. Peng XC, Gong FM, Zhao YW, Zhou LX, Xie YW, Liao HL, Lin HJ, Li ZY, Tang MH and Tong AP: Comparative proteomic approach identifies PKM2 and cofilin-1 as potential diagnostic, prognostic and therapeutic targets for pulmonary adenocarcinoma. PLoS One 6: e27309, 2011.

18. Li D, Zhang Y, Li Z, Wang X, Qu X and Liu Y: Activated Pak4 expression correlates with poor prognosis in human gastric cancer patients. Tumour Biol 36: 9431-9436, 2015.

19. Nishimura S, Tsuda H, Kataoka F, Arao T, Nomura H, Chiyoda T, Susumu N, Nishio K and Aoki D: Overexpression of cofilin 1 can predict progression-free survival in patients with epithelial ovarian cancer receiving standard therapy. Hum Pathol 42: 516-521, 2011.

20. Yang ZL, Miao X, Xiong L, Zou Q, Yuan Y, Li J, Liang L, Chen M and Chen S: CFL1 and Arp3 are biomarkers for metastasis and poor prognosis of squamous cell/adenosquamous carcinomas and adenocarcinomas of gallbladder. Cancer Invest 31: 132-139, 2013.
21. Wang W, Goswami S, Lapidus K, Wells AL, Wyckoff JB, Sahai E, Singer RH, Segall JE and Condeelis JS: Identification and testing of a gene expression signature of invasive carcinoma cells within primary mammary tumors. Cancer Res 64: 8585-8594, 2004.

22. Wang W, Mouneimne G, Sidani M, Wyckoff J, Chen X, Makris A, Goswami S, Bresnick AR and Condeelis JS: The activity status of cofilin is directly related to invasion, intravasation, and metastasis of mammary tumors. J Cell Biol 173: 395-404, 2006.

23. Shaheed SU, Rustogi N, Scally A, Wilson J, Thygesen H, Loizidou MA, Hadjisavvas A, Hanby A, Speirs V, Loadman P, et al: Identification of stage-specific breast markers using quantitative proteomics. J Proteome Res 12: 5696-5708, 2013.

24. Falck AK, Bendahl PO, Chebil G, Olsson H, Fernö M and Rydén L: Biomarker expression and St Gallen molecular subtype classification in primary tumours, synchronous lymph node metastases and asynchronous relapses in primary breast cancer patients with 10 years' follow-up. Breast Cancer Res Treat 140: 93-104, 2013

25. Rydén L, Jirström K, Bendahl PO, Fernö M, Nordenskjöld B, Stål O, Thorstenson S, Jönsson PE and Landberg G: Tumorspecific expression of vascular endothelial growth factor receptor 2 but not vascular endothelial growth factor or human epidermal growth factor receptor 2 is associated with impaired response to adjuvant tamoxifen in premenopausal breast cancer. J Clin Oncol 23: 4695-4704, 2005.

26. Edge S, Byrd DR, Compton CC, Fritz AG, Greene FL and Trotti A (eds): AJCC Cancer Staging Handbook. 7th edition. Springer, New York, NY, 2010.

27. Krishnamurthy S, Mathews K, McClure S, Murray M, Gilcrease M, Albarracin C, Spinosa J, Chang B, Ho J, Holt J, et al: Multi-institutional comparison of whole slide digital imaging and optical microscopy for interpretation of hematoxylin-eosin-stained breast tissue sections. Arch Pathol Lab Med 137: 1733-1739, 2013.

28. Rakha EA, Reis-Filho JS, Baehner F, Dabbs DJ, Decker T, Eusebi V, Fox SB, Ichihara S, Jacquemier J, Lakhani SR, et al: Breast cancer prognostic classification in the molecular era: The role of histological grade. Breast Cancer Res 12: 207, 2010.

29. Ono S: Mechanism of depolymerization and severing of actin filaments and its significance in cytoskeletal dynamics. Int Rev Cytol 258: 1-82, 2007.

30. van Rheenen J, Song X, van Roosmalen W, Cammer M, Chen X, Desmarais V, Yip SC, Backer JM, Eddy RJ and Condeelis JS: EGF-induced PIP2 hydrolysis releases and activates cofilin locally in carcinoma cells. J Cell Biol 179: 1247-1259, 2007.

31. Garg P, Verma R, Cook L, Soofi A, Venkatareddy M, George B, Mizuno K, Gurniak C, Witke W and Holzman LB: Actin-depolymerizing factor cofilin-1 is necessary in maintaining mature podocyte architecture. J Biol Chem 285: 22676-22688, 2010.

32. Tammana TV, Sahasrabuddhe AA, Bajpai VK and Gupta CM: $\mathrm{ADF} /$ cofilin-driven actin dynamics in early events of Leishmania cell division. J Cell Sci 123: 1894-1901, 2010.

33. Ellsworth RE, Hooke JA, Love B, Ellsworth DL and Shriver CD: Molecular changes in primary breast tumors and the nottingham histologic score. Pathol Oncol Res 15: 541-547, 2009.

34. Niikura N, Iwamoto T, Masuda S, Kumaki N, Xiaoyan T, Shirane M, Mori K, Tsuda B, Okamura T, Saito Y, et al: Immunohistochemical Ki67 labeling index has similar proliferation predictive power to various gene signatures in breast cancer. Cancer Sci 103: 1508-1512, 2012.

35. Volpi A, Nanni O, De Paola F, Granato AM, Mangia A, Monti F, Schittulli F, De Lena M, Scarpi E, Rosetti P, et al: HER-2 expression and cell proliferation: Prognostic markers in patients with node-negative breast cancer. J Clin Oncol 21: 2708-2712, 2003.

36. Mouneimne G, DesMarais V, Sidani M, Scemes E, Wang W, Song X, Eddy R and Condeelis J: Spatial and temporal control of cofilin activity is required for directional sensing during chemotaxis. Curr Biol 16: 2193-2205, 2006.

37. Oser M, Yamaguchi H, Mader CC, Bravo-Cordero JJ, Arias M, Chen X, Desmarais V, van Rheenen J, Koleske AJ and Condeelis J: Cortactin regulates cofilin and N-WASp activities to control the stages of invadopodium assembly and maturation. J Cell Biol 186: 571-587, 2009.

38. Huang X, Sun D, Pan Q, Wen W, Chen Y, Xin X, Huang M, Ding J and Geng M: JG6, a novel marine-derived oligosaccharide, suppresses breast cancer metastasis via binding to cofilin. Oncotarget 5: 3568-3578, 2014.

39. Zhang $\mathrm{Y}$ and Tong $\mathrm{X}$ : Expression of the actin-binding proteins indicates that cofilin and fascin are related to breast tumour size. J Int Med Res 38: 1042-1048, 2010. 
40. Polachini GM, Sobral LM, Mercante AM, Paes-Leme AF, Xavier FC, Henrique T, Guimarães DM, Vidotto A, Fukuyama EE, Góis-Filho JF, et al: Proteomic approaches identify members of cofilin pathway involved in oral tumorigenesis. PLoS One 7: e50517, 2012

41. Lu LI, Fu NI, Luo XU, Li XY and Li XP: Overexpression of cofilin 1 in prostate cancer and the corresponding clinical implications. Oncol Lett 9: 2757-2761, 2015.
42. Naumann DN and Sintler M: The surgeon as the most important factor in lymph node harvest during axillary clearance. Anticancer Res 33: 3935-3939, 2013.

43. Olivotto IA, Gomi A, Bancej C, Brisson J, Tonita J, Kan L, Mah Z, Harrison M and Shumak R: Influence of delay to diagnosis on prognostic indicators of screen-detected breast carcinoma. Cancer 94: 2143-2150, 2002. 